TRANSACTIONS OF THE

AMERICAN MATHEMATICAL SOCIETY

Volume 174, December 1972

\title{
ALGEBRAS OF ANALYTIC GERMS
}

BY

\author{
WILLIAM R. ZAME $\left({ }^{1}\right)$
}

\begin{abstract}
Let $S$ be a Stein-Riemann domain with global local coordinates $\sigma_{1}, \cdots, \sigma_{n}$. Let $X$ be a compact subset of $S$. Denote by $\mathcal{O}(X)$ the algebra of germs on $X$ of functions analytic near $X$. A subalgebra of $\mathcal{O}(X)$ containing the germs of $\sigma_{1}, \cdots, \sigma_{n}$ and the constants is stable if it is closed under differentiation with respect to the coordinates $\sigma_{1}, \cdots, \sigma_{n^{*}}$ In this paper the relationship of a stable algebra to its spectrum is investigated. In general, there is no natural imbedding of the spectrum into a Stein manifold. We give necessary and sufficient conditions that such an imbedding exists, and show that a stable algebra whose spectrum admits such an imbedding has a simple description. More generally, we show that a stable algebra is determined by its spectrum. This leads to certain approximation theorems.
\end{abstract}

1. Introduction. A central problem in the theory of several complex variables is that of determining which analytic functions can be approximated by analytic functions belonging to a particular algebra. An important case can be handled via the Oka-Weil theorem: if $K$ is a compact set in $\mathrm{C}^{n}$, then a function analytic near the polynomially convex hull of $K$ can be approximated near $K$ by polynomials. As the polynomially convex hull of $K$ can be identified with the spectrum of the algebra of polynomials on $K$, this suggests the importance of investigating the spectra of certain algebras of analytic functions.

The natural setting for this investigation is algebras of functions analytic near a compact subset $K$ of a Riemann domain. In order to avoid the complications that may arise when $K$ is relatively thin, it is convenient to consider germs of functions on $K$, rather than the restrictions to $K$ of these functions, and to endow the algebra of germs that arises with a natural topology; the spectrum is unaffected by these changes. The algebras we are primarily concerned with are closed under differentiation and contain the germs of all global analytic functions; we call such algebras stable. The completion of the algebra of germs of polynomials on a compact set in $\mathrm{C}^{n}$ certainly enjoys the se properties.

In $\$ 2$, we describe the general setup and collect some preliminary results. $\S 3$ is concerned with describing the spectrum of a stable algebra. Unlike the

Received by the editors November 4, 1971. $46 \mathrm{~J} 10$.

AMS (MOS) subject classifications (1970). Primary 32E25; Secondary 32E30, 32D15,

Key words and phrases. Riemann domains, germs of analytic functions, differentiably stable algebras, spectrum, approximation of analytic functions.

( $\left.{ }^{1}\right)$ The main results of this paper comprise a portion of the author's doctoral dissertation at Tulane University, written under the guidance of Professor Frank T. Birtel. The support of the National Science Foundation and Tulane University is gratefully acknowledged.

Copyright $\odot$ 1973, American Mathematical Society 
situation for the polynomials, the spectrum of a stable algebra cannot generally be realized as a subset of a complex manifold; we give necessary and sufficient conditions under which such a realization is possible. This leads to a nice description of such algebras. In $\$ 4$ we show that a stable algebra is determined by its spectrum and derive an approximation theorem which is analogous to the polynomial case. In $\$ 5$ we consider briefly algebras which do not have many global germs, and show that the study of such algebras may often be reduced to the study of stable algebras.

2. Definitions and preliminary results. Throughout this paper we let $S$ be a Ste in manifold of dimension $n$ with global local coordinate $\sigma=\left(\sigma_{1}, \cdots, \sigma_{n}\right)$; i.e., a Stein-Riemann domain. If $U$ is an open subset of $S$, then $O(U)$ denotes the algebra of functions analytic on $U$, equipped with the topology of uniform convergence on compact sets. For $V \subset U, r_{U V}: \Theta(U) \rightarrow \Theta(V)$ denotes the restriction. If $K$ is a compact subset of $S$, then $\left\{\Theta(U), r_{U V}: K \subset V \subset U\right\}$ is an inductive system of topological algebras; we let $\Theta(K)$ denote the inductive limit. Then $\Theta(K)$ is the algebra of germs on $K$ of functions analytic in some neighborhood of $K$. If $f$ is analytic near $K$, we denote the germ of $f$ on $K$ by $\mathrm{f}$; $f$ is a representative of $\mathrm{f}$. The map of $\Theta(U)$ into $\Theta(K)$ given by $f \rightarrow \mathrm{f}$ will be denoted by $r_{U}$. We give $\Theta(K)$ the inductive limit topology; i.e., the finest (not necessarily locally convex) topology rendering each $r_{U}$ continuous. This topology is described in the following proposition, the proof of which is a standard argument (see [6], [8], [12] for more detailed discussion).

Proposition 2.1. Let $\mathcal{F} \subset \mathcal{O}(K)$ and $\mathrm{f} \in \mathcal{O}(K)$. Then $\mathrm{f}$ lies in the closure of $\mathcal{F}$ if and only if there is an open set $U \supset K$ and functions $f, f_{1}, \ldots$, in $\Theta(U)$ sucb that $f$ is a representative of $f$, each $f_{i}$ is a representative of a germ in $\mathcal{F}$, and $f_{i}$ converges to $f$ in $\Theta(U)$.

If $A$ is a subalgebra of $\Theta(K)$ containing 1 , set $A(U)=r^{-1}(A)=\{f \in \Theta(U)$ : $\mathbf{f} \epsilon$ $A\}$ for each open $U$ containing $K$. It is easy to see that $\left\{A(U) ; r_{U V}\right\}$ is an inductive system and that (algebraically)

$$
A=\text { inductive limit }\left\{A(U) ; r_{U V}\right\}
$$

Using 2.1, it may be seen that the inductive limit topology on $A$ from the system $\left\{A(U) ; r_{U V}\right\}$ agrees with the subspace topology from $\mathcal{O}(K)$. (It is for this reason that we prefer the nonlocally convex inductive limit topology; the continuous linear functionals are identical, in any case.)

If $\mathrm{f} \in \mathcal{O}(K)$, we may abuse terminology and regard $\mathrm{f}$ as a function on $K$. We set

$$
\|\mathbf{f}\|_{K}=\sup \{|\mathbf{f}(x)|: x \in K\} .
$$


Then $\|\cdot\|_{K}$ is a continuous seminorm on $\Theta(K)$, and defines a (possibly non-Hausdorff) topology on $\Theta(K)$. The following proposition seems to have first been noticed by Harvey and Wells [10] for $O(K)$.

Proposition 2.2. Let $A$ be a subalgebra of $\Theta(K)$ containing 1. Then the inductive limit topology and the seminorm topology admit the same continuous complex-valued homomorphisms.

Proof. If this were not so, there would be a homomorphism $\phi$ of $A$, continuous relative to the inductive limit topology, and a germ $f \in A$ such that $\phi(f)=1>$ $\|\mathbf{f}\|_{K}$. We may choose an open set $U \subset K$ and a representative $f \in A(U)$ of $\mathbf{f}$ such that $\|f\|_{U}<1$. Then $1-f$ is invertible in $\Theta(U)$, and its inverse lies in the closure of $A(U)$. Since $\phi \circ r_{U}$ is a continuous homomorphism of $A(U)$, it extends to the closure. This yields the following contradictory chain of equalities:

$$
1=\phi \circ r_{U}\left[(1-f)^{-1}(1-f)\right]=\left\{\phi \circ r_{U}\left[(1-f)^{-1}\right]\right\}\left\{\phi \circ r_{U}(1-f)\right\}=0,
$$

since $\phi \circ r_{U}(1-f)=\phi(1-f)=0$. This contradiction establishes the proposition.

Observe from 2.1 that $A$ is closed in $\mathcal{O}(K)$ if and only if $A(U)$ is closed in $\Theta(U)$ for each $U$; thus the same argument may be used to establish the following proposition.

Proposition 2.3. If $A$ is a closed subalgebra of $\Theta(K)$ containing 1 , then every complex-valued bomomorphism of $A$ is continuous.

If $B$ is a topological algebra, then the spectrum $\Delta B$ of $B$ is the space of continuous, nonzero, complex-valued homomorphisms of $B$, equipped with the weak *-topology. If $f \in B$ then the Gelfand transform of $f$ is the continuous function $f^{\wedge}$ on $\Delta B$ defined by $f^{\wedge}(\phi)=\phi(f)$ for all $\phi \in \Delta B$.

If $A$ is a subalgebra of $\Theta(K)$ containing 1 , let $A_{0}$ denote the algebra of continuous functions on $K$ that agree with germs in $A$. The map $A \rightarrow A_{0}$ has as its kernel the collection of germs $\mathrm{f}$ such that $\|\mathrm{f}\|_{K}=0$. If we give $A_{0}$ the norm $\|\cdot\|_{K}$, then from 2.2 , together with the fact that $\|\mathrm{f}\|_{K}=0$ implies $\hat{f} \equiv 0$ on $\Delta A$, it follows that $A$ and $A_{0}$ have the same spectrum. Since $\Delta A_{0}$ is compact, this enables us to use techniques from the theory of function algebras. We refer to [7] for facts concerning function algebras.

If $U$ and $V$ are open with $K \subset V \subset U$, then the maps $r_{U V}, r_{U}$ have adjoints $r_{U V}^{*}: \Delta A(U) \rightarrow \Delta A(U)$ and $r_{U}^{*}: \Delta A \rightarrow \Delta A(U)$ given by

$$
r_{U V}^{*}(\phi)(f)=\phi(f \mid V) ; \quad r_{U}^{*}(\phi)(f)=\phi(f) .
$$

A straightforward argument similar to the locally convex case shows that

$$
\Delta A=\text { projective limit }\left\{\Delta A(U) ; r_{U V}^{*}\right\}
$$

and the maps $r_{U}^{*}$ are projective limit maps.

Let $D_{j}$ denote differentiation with respect to $\sigma_{j}$. For each $j$ and each open 
set $U, D_{j}$ is a continuous linear operator on $\Theta(U)$. Since $D_{j}$ commutes with the restrictions $r_{U V}$, there is induced on $\Theta(K)$ a continuous linear operator $D_{j}$ commuting with the restrictions $r_{U}$.

Definition 2.4. Let $K$ be a compact subset of $S$. A subalgebra $A$ of $\Theta(K)$ will be called stable if

(i) $\mathbf{f} \in A$ for each $f \in \mathcal{O}(S)$;

(ii) $D_{j} f \in A$ for each $\mathbf{f} \in A, 1 \leq j \leq n$.

Condition (i) ensures that $A$ contains "enough" germs; in particular, $\sigma_{i} \in A$ for each $i$, and $A$ separates the points of $K$. (In $\$ 5$, we examine the effect of weakening this condition.) Observe that (ii) is equivalent to the requirement that each $A(U)$ be closed under differentiation.

The following result is due to Bishop and is essentially contained in [4].

Theorem 2.5. Let $M$ be a Riemann domain with global local coordinate $\mu=$ $\left(\mu_{1}, \cdots, \mu_{n}\right)$ and let $B$ be a closed subalgebra of $\Theta(M)$ containing the $\sigma_{i}$ and the constants, and closed under differentiation. Then $\Delta B$ may be given the structure of a Stein-Riemann domain in such a way that:

(i) $\hat{\mu}=\left(\hat{\mu_{1}}, \cdots, \hat{\mu_{n}}\right)$ is a global local coordinate;

(ii) $B^{\wedge}=\Theta(\Delta B)$;

(iii) the map $\delta: M \rightarrow \Delta B$ given by $\delta(m)(f)=f(m)$ is an analytic local bomeomorphism; it is a bomeomorphism if $B$ separates the points of $M$;

(iv) every component of $\Delta B$ intersects $\delta(M)$;

(v) for each $f \in B$ and each $j,\left(D_{j} f\right)^{\hat{}}=D_{j}(\hat{f})$, where $D_{j}(\hat{f})$ denotes the derivative of $\hat{f}$ with respect to $\hat{\sigma_{j}}$.

Returning to our situation, since $S$ is Stein, $\Delta \Theta(S)=S$. If we let $\pi(\phi)=$ $\left(\phi\left(\sigma_{1}\right), \cdots, \phi\left(\sigma_{n}\right)\right)$ and $\pi_{U}(\phi)=\left(\phi\left(\sigma_{1} \mid U\right), \cdots, \phi\left(\sigma_{n} \mid U\right)\right)$, then we have the commutative diagram of Figure 1 , where $\Delta A(U), \Delta A(V), S$ and $\mathrm{C}^{n}$ are Stein-Riemann domains and the maps between them are analytic local homeomorphisms.

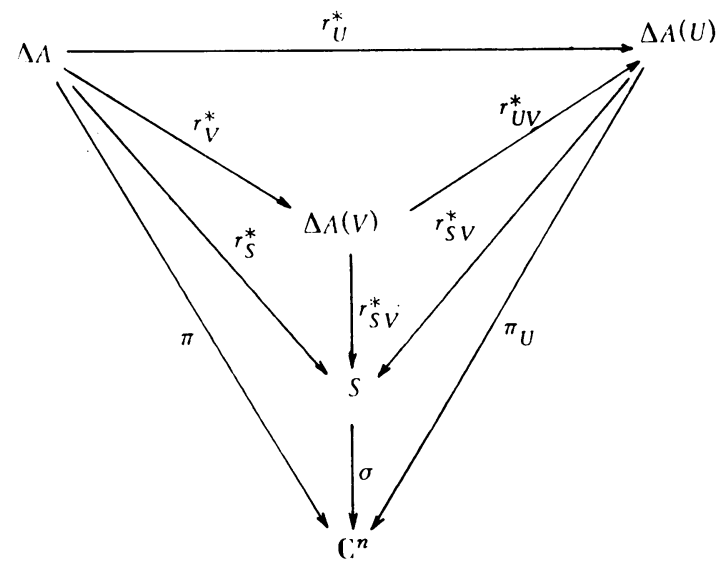

Figure 1 
3. Geometry of the spectrum. Let $K$ be a compact subset of $S$ and $A$ a stable subalgebra of $\Theta(K)$. In investigating the spectrum of $A$, there is clearly no loss of generality in assuming $A$ to be closed in $\mathcal{O}(K)$. We retain the notation at the end of $\$ 2$; in particular we refer to the commutative diagram of Figure 1.

Proposition 3.1. If $x$ is a point of $\pi(\Delta A)$, then $\pi^{-1}(x)$ is totally disconnected.

Proof. Let $F$ be a connected component of $\pi^{-1}(x)$ and suppose that $p$ and $q$ are distinct points of $F$. We can find an open set $U$ containing $K$ such that $r_{U}^{*}(p) \neq r_{U}^{*}(q)$. Observe that $r_{U}^{*}(F)$ is connected and that $\pi_{U} \circ r_{U}^{*}(F)=\pi(F)=\{x\}$. Since $\pi_{U}$ is a local homeomorphism, there is an open set $W$ in $\Delta A(U)$ which contains $r_{U}^{*}(p)$, and on which $\pi_{U}$ is a homeomorphism. But then $r_{U}^{*}(F) \cap W=\left\{r_{U}^{*}(p)\right\}$, which is absurd in view of the connectedness of $r_{U}^{*}(F)$. It follows that $F$ is a singleton, so that $\pi^{-1}(x)$ is totally disconnected.

Corollary 3.2. The topological dimension of $\Delta A$ does not exceed $2 n$.

Proof. By 3.1, the fibers $\pi^{-1}(x)$ have dimension zero; since $\pi$ is a closed mapping, it follows from Theorem III. 6 of [14] that the dimension of $\Delta A$ does not exceed the dimension of $\pi(\Delta A)$.

Now we give necessary and sufficient conditions under which $\Delta A$ admits a "nice" imbedding into a complex manifold. When such an imbedding exists, there is an imbedding into $\Delta A(U)$ for some open $U$ containing $K$; then $A$ may be realized as $\mathcal{O}\left(r_{U}^{*}(\Delta A)\right)$. First we need a lemma, the proof of which is a standard compactness argument.

Lemma 3.3. If $X$ and $Y$ are disjoint closed subsets of $\Delta A$ then there is an open set $U$ containing $K$ such that $r_{U}^{*}(X)$ and $r_{U}^{*}(Y)$ are disjoint.

Theorem 3.4. The following conditions are equivalent:

(a) $\pi$ is locally one-to-one;

(b) $r_{S}^{*}$ is locally one-to-one;

(c) there is an open set $U$ containing $K$ such that $r_{U}^{*}$ maps $\Delta A$ bomeomorpbically into $\Delta A(U)$;

(d) there is a Riemann domain $M$ of some dimension $k$ and with spread map $\mu=\left(\mu_{1}, \cdots, \mu_{k}\right)$, and a bomeomorphism $F$ of $\Delta A$ into $M$ sucb that $\mu_{i} \circ F \in A^{\wedge}$ for each $i$;

(e) there is a Stein manifold $N$ and a bomeomorphism $G$ of $\Delta A$ into $N$ such that $b^{\circ} \circ \in A^{\wedge}$ for eacb $b \in \mathcal{C}(N)$.

Proof. Since $\pi=r_{S}^{*} \circ \sigma$ and $\sigma$ is a local homeomorphism, it is clear that (a) and (b) are equivalent. It is easy to see that (c) implies (d) and (e) since $\Delta A(U)$ is a Stein-Riemann domain. We show that (a) implies (c), (d) implies (a) and (e) implies (c). 
Assume that for each point $p \in \Delta A$ there is an open set $W(p)$ on which $\pi$ is one-to-one. Let $x$ be a point of $\pi(\Delta A)$. Since $\pi^{-1}(x)$ is compact, we may choose finitely many points $p_{1}, \cdots, p_{k}$ in $\pi^{-1}(x)$ such that $\pi^{-1}(x) \subset \bigcup W\left(p_{i}\right)$. We see that $W\left(p_{i}\right) \cap \pi^{-1}(x)=\left\{p_{i}\right\}$. Thus there are open sets $W^{\prime}\left(p_{i}\right)$ such that $p_{i} \in W^{\prime}\left(p_{i}\right) \subset$ $W^{\prime}\left(p_{i}\right)^{-} \subset W\left(p_{i}\right)$ for each $i$. Then for $i \neq j, W^{\prime}\left(p_{i}\right)^{-} \cap W^{\prime}\left(p_{j}\right)^{-}=\varnothing$. Repeated applications of 3.3 show that there is an open set $U(x)$ containing $K$ such that

$$
r_{U(x)}^{*}\left[W^{\prime}\left(p_{i}\right)^{-}\right] \cap r_{U(x)}^{*}\left[W^{\prime}\left(p_{j}\right)^{-}\right]=\varnothing
$$

whenever $i \neq j$.

Choose an open set $V(x)$ in $\pi(\Delta A)$ containing $x$ for which

$$
\pi^{-1}(V(x)) \subset \bigcup W^{\prime}\left(p_{i}\right)
$$

this is possible since $U W^{\prime}\left(p_{i}\right)$ is a neighborhood of $\pi^{-1}(x)$ in $\Delta A$. Let $s$ and $t$ be distinct points of $\pi^{-1}(V(x))$ and suppose that $s \in W^{\prime}\left(p_{i}\right)$ and $t \in W^{\prime}\left(p_{j}\right)$. If $i \neq$ $j$ then our construction assures us that $r_{U(x)}^{*}(s) \neq r_{U(x)}^{*}(t)$; if $i=j$ we already have this condition.

Thus for each $x$ in $\Delta A$ we have found open sets $U(x)$ and $V(x)$ with $x \in V(x) C$ $\Delta A$ and $K \subset U(x) \subset S$ such that if $s$ and $t$ are distinct points of $\pi^{-1}(V(x))$ then $r_{U(x)}^{*}(s) \neq r_{U(x)}^{*}(t)$. Compactness of $\Delta A$ insures that we may find finitely many points $x_{1}, x_{2}, \cdots, x_{m}$ in $\pi(\Delta A)$ such that $V\left(x_{1}\right), \cdots, V\left(x_{m}\right)$ cover $\pi(\Delta A)$. If we let $U=\bigcap U\left(x_{i}\right)$ then it is easy to see that $r_{U}^{*}$ maps $\Delta A$ homeomorphically into $\Delta A(U)$. Thus (a) implies (c).

To see that (d) implies (a), choose germs $\mathrm{f}_{1}, \cdots, \mathrm{f}_{k}$ in $A$ such that $\mu_{i} \circ F=$ $\hat{f_{i}^{\wedge}}$ for each $i$. We may find an open set $U$ containing $K$ and functions $f_{1}, \cdots, f_{k}$ in $A(U)$ representing $\mathrm{f}_{1}, \cdots, \mathrm{f}_{k}$ respectively. Let $H=\left(\hat{f_{1}}, \cdots, \hat{f_{k}}\right)$ and $J=$ $\left(\mathrm{f}_{1}^{\wedge}, \cdots, \mathrm{f}_{k}^{\wedge}\right)$. Then $J=\mu \circ F=H \circ r_{U}^{*}$. If $p \in \Delta A$ then there is an open set $W$ such that $F(p) \in W \subset M$ and $\mu$ is a homeomorphism on $W$. Thus, $H \circ r_{U}^{*}$ is one-toone on the open set $F^{-1}(W) \subset \Delta A$. There is an open set $W^{\prime} \subset \Delta A(U)$ containing $r_{U}^{*}(p)$ on which $\pi_{U}$ is a homeomorphism. If we set $Q=r_{U}^{*-1}\left(W^{\prime}\right) \cap F^{-1}(W)$, we may see that $Q$ is an open subset of $\Delta A$ containing $p$ on which $\pi$ is one-to-one.

Finally we show that (e) implies (c). It is well known (see [9] for example) that there are a finite number of functions $b_{1}, \cdots, b_{r}$ in $\Theta(N)$ such that $H=$ $\left(b_{1}, \cdots, b_{r}\right)$ is an analytic isomorphism of $N$ with a closed submanifold of $\mathbf{C}^{r}$. There are germs $\mathbf{g}_{1}, \cdots, \mathbf{g}_{r}$ in $A$ such that $b_{i} \circ G=\mathbf{g}_{i}$ for each $i$. Set $H^{\prime}=$ $\left(\mathrm{g}_{1}, \cdots, \hat{\mathbf{g}_{r}}\right)$ and observe that $H \circ G=H^{\prime} \circ r_{U}^{*}$. It follows that $r_{U}^{*}$ is one-to-one, which completes the proof.

Lemma 3.5. Let $U$ be an open set containing $K$ and $W$ a neighborbood of $r_{U}^{*}(\Delta A)$ in $\Delta A(U)$. Then there is an open set $V$ with $K \subset V \subset U$ such that $r_{U V}^{*}(\Delta A(V)) \subset W$.

Proof. Choose a fundamental system $\left\{U_{i}\right\}$ of relatively compact neighborhoods 
of $K$ such that $U_{i+1}^{-} \subset U_{i} \subset U$. For each $i$, let $X_{i}$ be the $A\left(U_{i}\right)$-hull of $U_{i+1}^{-}$; that is

$$
X_{i}=\left\{\phi \in \Delta A\left(U_{i}\right):|\phi(f)| \leq \sup |f(x)|\right\}
$$

where the supremum extends over all $x \in U_{i+1}^{-}$and all $f \in A\left(U_{i}\right)$. Since $\Delta A\left(U_{i}\right)$ is Stein, $X_{i}$ is compact. It is easy to see that

$$
K \subset r_{U_{i+1}}^{*}\left(\Delta A\left(U_{i+1}\right)\right) \subset X_{i}
$$

and that $\Delta A$ is the projective limit of the system $\left\{X_{i} ; r_{U_{j} U_{i}}^{*}\right\}$. Standard arguments on the projective limit of compact spaces show that there ${ }^{j}{ }^{i}$ an integer $k$ such that $r_{U U_{k}}^{*}\left(X_{k}\right) \subset W$. Then we certainly have $r_{U U_{k+1}}^{*}\left(\Delta A\left(U_{k+1}\right)\right) \subset W$ as desired.

Theorem 3.6. Let $U$ be an open set containing $K$ and suppose that $r_{U}^{*}$ is a bomeomorphism of $\Delta A$ into $\Delta A(U)$. Then $A=\mathcal{O}\left(r_{U}^{*}(\Delta A)\right)$.

Proof. Let $\mathrm{f}$ be a germ in $\Theta\left(r_{U}^{*}(\Delta A)\right)$ and let $f \in \mathcal{O}(W)$ represent $\mathrm{f}$. We may choose an open set $V$ with $K \subset V \subset U$ such that $f_{U V}^{*}(\Delta A(V)) \subset W$. Then $f \circ r_{U V}^{*}$ is an analytic function on $\Delta A(V)$ and is thus the transform of a function $f_{1} \epsilon$ $A(V)$. A straightforward calculation shows that $\mathrm{f}_{1}$ is independent of the choice of representative for $f$ and the open set $V$. Thus if we set $\Phi(\mathfrak{f})=f_{1}$, it may be seen that $\Phi$ is well defined and is a continuous homomorphism of topological algebras. We show that it is an isomorphism.

Let $\mathbf{f} \epsilon A$ and choose an open set $V, K \subset V \subset U$, and a representative $f \epsilon$ $A(V)$. Since $r_{U}^{*}$ is a homeomorphism and $r_{U}^{*}=r_{U V}^{*} \circ r_{V}^{*}$, it follows that $r_{U V}^{*}$ maps $r_{U}^{*}(\Delta A)$ homeomorphically onto $r_{U}^{*}(\Delta A)$. Then there is an open set $Q \supset r_{V}^{*}(\Delta A)$ such that $r_{U V}^{*}$ maps $Q$ homeomorphically onto the open set $Q^{\prime}$. Let $f_{2}=f^{\wedge}$ 。 $\left[r_{U V}^{*} \mid Q\right]^{-1}$, so that $f_{2} \in \mathcal{O}\left(Q^{\prime}\right)$. If we let $f_{2}$ be the germ of $f_{2}$ on $r_{U}^{*}(\Delta A)$, it is easy to see that $\Phi\left(f_{2}\right)=f$. Hence $\Phi$ is onto.

If $f$ and $g$ are distinct germs in $\mathcal{O}\left(r_{U}^{*}(\Delta A)\right)$, then they define different functions on each of the manifolds $\Delta A(V)$. Thus $\Phi(\mathrm{f}) \neq \Phi(\mathrm{g}) ; \Phi$ is one-to-one.

It remains to show that $\Phi^{-1}$ is continuous. To this end, suppose that $\left\{g_{i}\right\}$ is a sequence of germs in $A$ tending to 0 . Then there is an open set $V$ containing $K$ and representatives $g_{i} \in A(V)$ which tend to 0 uniformly on compact subsets of $V$. With the aid of the above construction, it may be seen that $\hat{g_{i}} \circ\left[r_{U V}^{*} \mid Q\right]^{-1}$ tends to 0 uniformly on compact subsets of $Q^{\prime}$. Thus $\Phi^{-1}\left(\mathbf{g}_{i}\right)$ tends to 0 in $\mathcal{O}\left(r_{U}^{*}(\Delta A)\right)$, which completes the proof.

If we regard $K$ as a subset of $\Delta A(U)$, and $A$ as an algebra of germs on $\Delta A(U)$, then the above proof shows that (under the hypotheses of 3.6) each germ in $A$ extends uniquely to a germ on $r_{U}^{*}(\Delta A)$.

In general, $\Delta A$ does not admit a natural imbedding into a manifold, and $A$ is not isomorphic to $\Theta(L)$ for any compact $L$, as the following example demonstrates. In $C$, let $\Gamma_{n}=\{z:|z|=1-1 / n\}$ for each $n>0$ and let $\Gamma_{0}=\{z:|z|=1\}$. Let 
$X=\bigcup_{n=0}^{\infty} \Gamma_{n}$ and let $A_{0}=\left\{f \in \mathcal{O}(X): \mathrm{f} \mid \Gamma_{n}\right.$ is a polynomial for each $\left.n\right\}$; let $A$ be the completion of $A_{0}$ in $\mathcal{O}(X)$. Then $A$ is a complete stable algebra on $X$. If $D_{n}$ is the closed disk bounded by $\Gamma_{n}$, then $\Delta A$ may be identified with the disjoint union of the $D_{n}$, topologized so that $D_{n}$ converges to $D_{0}$. Alternatively, we may identify $\Delta A$ with the set

$$
Y=\left\{(z, w) \in C^{2}:|z| \leq 1-w, w=1,1 / 2, \cdots 1 / n, \cdots, 0\right\} 。
$$

The equivalent conditions of 3.4 clearly fail here, and it is easy to see that $A$ is not isomorphic to $\Theta(L)$ for any compact $L$. (Observe that $\mathbf{w} \in \Theta(Y)$ but $w$ is not the transform of a germ in $A$.) Finally we observe that $\Theta(Y)$ and $A^{\wedge}$ have the same uniform completions as algebras of functions on $Y$.

Theorem 3.7. If $r_{S}^{*}(\Delta A)=K$, then $\Delta A=K$ and $A=\mathcal{C}(K)$.

Proof. If $\Delta A \neq K$, then there are points $x \in K$ and $y \in \Delta A$ such that $r_{S}^{*}(x)=$ $r_{S}^{*}(y)=x$ and $y \neq x$. For each $p \in \Delta A$, let $E(p)$ be the component of $\Delta A$ containing $p$. By the Šilov idempotent theorem, there is a point $q \in E(y) \cap K$. Now we have $r_{S}^{*}(q)=q \in r_{S}^{*}(E(y)), x=r_{S}^{*}(y) \in r_{S}^{*}(E(y))$, so that $r_{S}^{*}(E(y)) \subset E(x)$. It follows that $E(y)=E(x)=E(q)$. Choose an open set $U$ containing $K$ such that $r_{U}^{*}(x) \neq$ $r_{U}^{*}(y)$. Regarding $U$ as an open subset of $\Delta A(U)$, we see that $r_{U}^{*}(E(x))$ is compact, connected and meets both $U$ and $(\Delta A(U)-U)$. It follows that $r_{U}^{*}(E(x))$ contains a point of the boundary of $U$ relative to $\Delta A(U)$. Since $r_{S U}^{*} \circ r_{U}^{*}(E(x)) \subset K$, this is impossible. We conclude that $\Delta A=K$; it follows from 3.6 that $A=\mathcal{O}(K)$.

4. Characterization of stable algebras. In $\$ 3$ we arrived at a concise description of complete stable algebras with a "reasonable" spectrum. Such a description is not available for algebras whose spectrum is not well behaved, but we show that a complete stable algebra is determined by its spectrum. This leads to certain approximation results.

Theorem 4.1. Let $A$ and $B$ be complete stable algebras of germs on $K$ with $A \subset B$. Let $j: A \rightarrow B$ be the inclusion and $j^{*}: \Delta B \rightarrow \Delta A$ be its adjoint. If $j^{*}$ is a bomeomorphism, then $A=B$.

Proof. Choose and fix an open set $U$ containing $K$. We will show that $A(U)=$ $B(U)$.

Let $j_{U}: A(U) \rightarrow B(U)$ be the inclusion and $j_{U}^{*}$ be its adjoint. Let us use $\pi$, $\pi_{U}, r_{U}^{*}$ for the maps arising from $A$, and $\tau, \tau_{u}, \rho_{u}^{*}$ for the maps arising from $B$. Then we have the commutative diagram of Figure 2. 


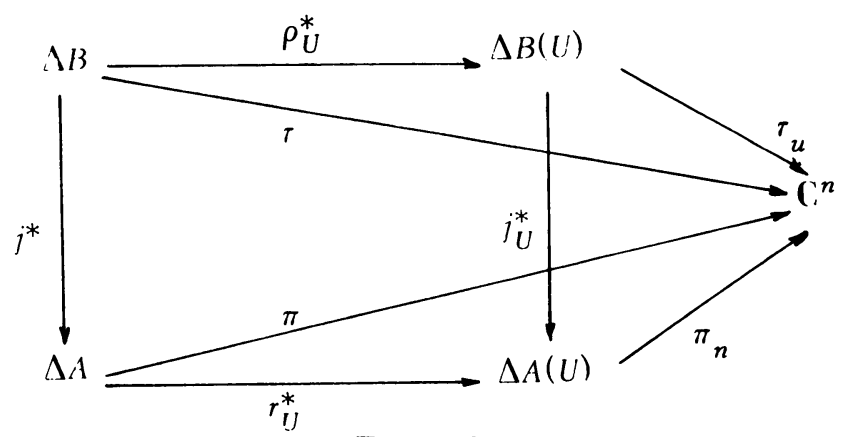

Figure 2

For each $x \in \Delta A$ define

$$
E(x)=j^{*} \circ \rho_{U}^{*-1} \circ \rho_{U}^{*} \circ j^{*-1}(x)=\rho_{U}^{*-1} \circ j_{U}^{*-1} \circ j_{U}^{*} \circ r_{U}^{*}(x) .
$$

The collection $\{E(x)\}$ is a decomposition of $\Delta A$ into disjoint compact sets. Observe that $\pi(E(x))=\pi(x)$. Set $Y=\pi(\Delta A)=\pi \circ j^{*}(\Delta B)=\tau(\Delta B)$ and let $y \in Y$. The set $\pi_{U}^{-1}(y) \cap \rho_{U}^{*}(\Delta B)$ is compact; since $\pi_{U}$ is a local homeomorphism it is also discrete, and hence finite. It follows that $\{E(x): \pi(x)=y\}$ is a finite collection.

Using 3.3 repeatedly, we can find an open set $W(y)$ about $K$ such that

$$
r_{W(y)}^{*}\left(E\left(x_{1}\right)\right) \cap r_{W(y)}^{*}\left(E\left(x_{2}\right)\right)=\varnothing
$$

provided that $\pi\left(x_{1}\right)=\pi\left(x_{2}\right)=y$, and that $E\left(x_{1}\right) \cap E\left(x_{2}\right)=\varnothing$. That is, the map $r_{W(y)}^{*}$ preserves the decomposition of $\pi^{-1}(y)$ into disjoint sets.

Let us write

$$
\tau_{U}^{-1}(y) \cap \rho_{U}^{*}(\Delta B)=\left\{b_{1}, \cdots, b_{s}\right\}
$$

For each $i$ choose an open set $Q_{i}$ in $\Delta B(U)$ which contains $b_{i}$ and is mapped homeomorphically by $\tau_{U}$ into a polydisc with center $y$. Since $s$ is finite, we may assume that all $Q_{i}$ are mapped onto the same polydisk, say $P(y)$. Set $Q=\bigcup Q_{j}$. Then $\rho_{U}^{*}(\Delta B)-Q$ is compact; so $\tau_{U}\left(\rho_{U}^{*}(\Delta B)-Q\right)$ is compact and misses $y$. By shrinking $Q_{i}$ and $P(y)$ as necessary, we may assume that

$$
P(y) \cap \tau_{U}\left(\rho_{U}^{*}(\Delta B)-Q\right)=\varnothing .
$$

We then have

$$
\tau_{U}^{-1}(P(y)) \cap \rho_{U}^{*}(\Delta B)=\bigcup\left[Q_{i} \cap \rho_{U}^{*}(\Delta B)\right]
$$

For each $i$, let $V_{i}=j^{*} \circ \rho_{U}^{*-1}\left(Q_{i}\right)$ so that the $V_{i}$ are disjoint open sets and $V_{i}$ contains $E\left(x_{i}\right)$ for each $i$. By shrinking $Q_{i}$ and $P(y)$ as necessary, we can effect a shrinking of the $V_{i}$ so that

$$
r_{W(y)}^{*}\left(V_{i}\right) \cap r_{W(y)}^{*}\left(V_{j}\right)=\varnothing
$$

whenever $i \neq j$. For each $p \in P(y)$, write 


$$
\tau_{U}^{-1}(p) \cap \rho_{U}^{*}(\Delta B)=\left\{d_{1}, \cdots, d_{t}\right\}
$$

Using the above facts, it follows that $t \leq s$. By a suitable renumbering, we may assume $d_{j} \in Q_{j}$ for $1 \leq j \leq t$. Let $x \in j^{*} \circ \rho_{U}^{*-1}\left(d_{1}\right)$. Then $x \in V_{1}$ and we have

$$
\rho_{U}^{*} \circ j^{*-1}(E(x))=\rho_{U}^{*} \circ j^{*-1}(x)=\left\{d_{1}\right\}
$$

so that $E(x) \subset V_{1}$ also. Furthermore,

$$
\pi^{-1}(p)=\bigcup\left[j * \circ \rho_{U}^{*-1}\left(d_{i}\right)\right]=\bigcup\left[\left(j^{*} \circ \tau^{-1}(p)\right) \cap V_{i}\right]=\bigcup E\left(x_{i}\right)
$$

where each $x_{i}$ is a point of $\left(j^{*} \circ \tau^{-1}(p)\right) \cap V_{i}$ and the unions extend over the indices $\mathrm{l} \leq i \leq t$.

Thus for each $y \in Y$, we have found an open set $W(y)$ containing $K$ and a polydisk $P(y)$ containing $y$ such that: if $a$ and $b$ are in $\Delta A$ with $\pi(a)=\pi(b) \in P(y)$, and $E(a) \cap E(b)=\varnothing$, then

$$
\left[r_{W(y)}^{*}(E(a))\right] \cap\left[r_{W(y)}^{*}(E(b))\right]=\varnothing .
$$

Observe that if $W$ is any open set with $K \subset W \subset W(y)$ then $r_{W}^{*}$ also enjoys the property of separating $E(a)$ from $E(b)$ whenever $\pi(a)=\pi(b) \in P(y)$ and $E(a) \cap E(b)=\varnothing$.

Since $Y$ is compact, we may choose a finite number of points $y_{1}, \cdots, y_{m}$ so

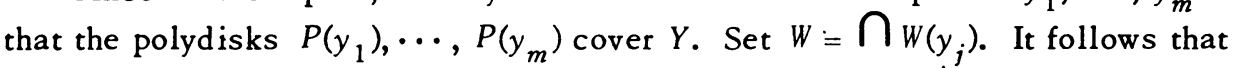
if $a, b$ are in $\Delta A$ with $\pi(a)=\pi(b)$, but $E(a) \cap E(b)=\varnothing$ then $r_{W}^{*}$ separates $E(a)$ from $E(b)$. On the other hand, if $\pi(a) \neq \pi(b)$ then $r_{W}^{*}$ automatically separates $E(a)$ from $E(b)$.

Now if $x$ and $y$ are in $\Delta B$ and $\rho_{U}^{*}(x)=\rho_{U}^{*}(y)$ then

$$
\left\{\rho_{W}^{*} \circ j^{*-1}\left[E\left(j^{*}(x)\right)\right]\right\} \cap\left\{\rho_{W}^{*} \circ j^{*-1}\left[E\left(j^{*}(y)\right)\right]\right\}=\varnothing .
$$

Thus if $p$ and $q$ are $\rho_{W}^{*}(\Delta B)$ and $j_{W}^{*}(p)=j_{W}^{*}(q)$, it follows that $(f \mid W)^{\wedge}(p)=$ $(f \mid W)^{\wedge}(q)$ for each $f \in B(U)$, since $f^{\wedge}$ is constant on the sets $j^{*-1}(E(x))$. We show that this property persists in a neighborhood of $\rho_{W}^{*}(\Delta B)$.

Let $p \in r_{W}^{*}(\Delta A)$ and choose a polydisk $P(p)$ about $\pi_{W}(p)$ and an open set $Q(p)$ about $p$ in $\Delta A(W)$ such that $\pi_{W}$ maps $Q(p)$ homeomorphically onto $P(p)$. Since $j_{W}^{*}$ is a local homeomorphism, $j_{W}^{*-1}(p) \cap \rho_{W}^{*}(\Delta B)$ is finite, equal to $\left\{q_{1}\right.$, $\cdots, q_{s}$ say. As in the first part of the proof, we may choose open sets $V_{1}(p)$, $\cdots, V_{s}(p)$ (shrinking $Q(p)$ and $P(p)$ as necessary) so that $q_{i} \in V_{i}(p), j_{W}^{*}$ maps $V_{i}(p)$ homeomorphically onto $Q(p)$ and

$$
j_{W}^{*-1}(Q(p)) \cap \rho_{W}^{*}(\Delta B)=\bigcup\left[\rho_{W}^{*}(\Delta B) \cap V_{i}(p)\right] .
$$

If we do this for each $p \in r_{W}^{*}(\Delta A)$, then the collection $\{Q(p)\}$ forms an open cover of $r_{W}^{*}(\Delta A)$; hence we can find a finite number of points, say $p_{1}, \cdots, p_{r}$, such that $Q\left(p_{1}\right), \cdots, Q\left(p_{r}\right)$ cover $r_{W}^{*}(\Delta A)$. For each $i$, let $s(i)$ denote the cardinality of the finite set $j_{W}^{*-1}\left(p_{i}\right) \cap \rho_{W}^{*}(\Delta B)$, and set 


$$
\Omega_{1}=\bigcup_{k=1}^{r} \bigcup_{m=1}^{s(k)} V_{m}\left(p_{k}\right), \quad \Omega_{2}=\bigcup_{k=1}^{r} Q\left(p_{k}\right) \text {. }
$$

Observe that $\Omega_{1}, \Omega_{2}$ are open sets and that $j_{W}^{*}\left(\Omega_{1}\right)=\Omega_{2}$.

Let $x, y \in \Omega_{1}$ with $j_{W}^{*}(x)=j_{W}^{*}(y)$ and let $f \in B(U)$. We assert that $(f \mid W)^{\wedge}(x)=$ $(f \mid W)^{\wedge}(y)$. In order to see this, first observe that there are integers $k, m$ and $m^{\prime}$ with $j_{W}^{*}(x)=j_{W}^{*}(y) \in Q\left(P_{k}\right), x \in V_{m}\left(p_{k}\right)$ and $y \in V_{m},\left(p_{k}\right)$. If $m=m^{\prime}$, then $x=y$ since $j_{W}^{*}$ is a homeomorphism on $V_{m}\left(p_{k}\right)$, so assume $m \neq m^{\prime}$. We can find points $q, q^{\prime} \in r_{W}^{*}(\Delta B)$ such that $q \in V_{m}\left(p_{k}\right), q^{\prime} \in V_{m}\left(p_{k}\right)$ and $j_{W}^{*}(q)=j_{W}^{*}\left(q^{\prime}\right)$. Then $(g \mid W)^{\wedge}(q)=(g \mid W)^{\wedge}\left(q^{\prime}\right)$ for each $g \in B(U)$; since $B(U)$ is closed under differentiation, it follows that $(f \mid W)^{\wedge}$ and all its derivatives agree at $q$ and $q^{\prime}$. Hence $(f \mid W)^{\wedge}(x)=(f \mid W)^{\wedge}(y)$ as asserted.

Now choose and fix $g \in B(U)$. Define a function $b$ on $\Omega_{2}$ by $b=g$ 。 $\left(j_{W}^{*} \mid \Omega_{1}\right)^{-1}$. The preceding paragraph shows that $b$ is well defined and analytic on $\Omega_{2}$. Since $\Omega_{2}$ contains $r_{W}^{*}(\Delta A)$, we can find an open set $W^{\prime}$ containing $K$ such that $r_{W W^{\prime}}^{*}\left(\Delta A\left(W^{\prime}\right)\right) \subset \Omega_{2}$. Then $H=b^{\circ} r^{*} W^{\prime}$ is analytic on $\Delta A\left(W^{\prime}\right)$. Regarding $W^{\prime}$ as an open subset of $S$ and of $\Delta A\left(W^{\prime}\right)$ we see that $b_{1}=g$ on $W^{\prime}$, so that $h_{1}=$ $\mathrm{g} \in A$. Thus $g \in A(U)$, and we have the desired result.

If we drop the requirement that $A$ and $B$ be complete, a slight modification of the above proof yields the following approximation theorem.

Theorem 4.2. Let $A$ and $B$ be stable algebras of germs on $K$ with $A \subset B$. If $j^{*}$ is a bomeomorphism of $\Delta B$ onto $\Delta A$ and $U$ is an open set containing $K$, then there is an open set $V$ with $K \subset V \subset U$ such that every function in $B(U) \mid V$ is the limit, uniformly on compact sets of functions in $A(V)$.

In [5], Bjork obtained independently the result that if $K$ is a compact set in $\mathrm{C}^{n}$ and $U$ is open and contains $K$, then there is an open $V, K \subset V \subset U$, such that every function $f \in \mathcal{Q}(U)$, approximable in a neighborhood of $K$ by polynomials, can be approximated on $V$ by polynomials. The following corollary to Theorem 4.2 is a more general result of this nature.

Corollary 4.3. Let $A$ be a stable algebra of germs on $K$ and let $U$ be an open set containing $K$. Let $E$ be the set of $f \in \mathcal{O}(U)$ such that $f$ and all its derivatives can be approximated uniformly on $K$ by germs in $A$. Then there is an open set $V$ with $K \subset V \subset U$ such that every function in $E$ can be approximated uniformly on compact subsets of $V$ by functions in $A(V)$.

It seems natural to ask whether the results of this section can be extended to nonstable algebras. For example, if $A, B$ are complete subalgebras of $\Theta(K)$, $A$ is stable and $\Delta A=\Delta B$, does it follow that $A=B$ ? In [13], it was shown that this is the case when $K \subset C^{1}$. The following example, suggested to the author by Eva Kallin, shows that this is not the case in higher dimensions. 
We use Eva Kallin's nonlocal function algebra [15]. Let $K$ be the compact polynomially convex set there described, for which $P(K)$, the algebra of uniform limits of polynomials on $K$, is not local. The function $f$ which is locally in $P(K)$ but not in $P(K)$ is analytic in a neighborhood of bdy $K$. Take $A=$ completion in

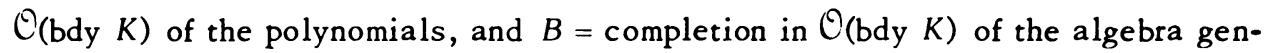
erated by $A$ and $\mathrm{f}$. Then $\Delta A=\Delta B=K$ but $A \neq B$. The essential difficulty is that the elements of $A$ extend to be analytic near $K$ while $f$ does not.

5. Algebras with few global germs. Let $A$ be a closed subalgebra of $\mathcal{O}(K)$ containing the germs $1, \sigma_{1}, \cdots, \sigma_{n}$, and closed under differentiation. One may ask whether $A$ is isomorphic to a stable algebra. An affirmative solution is easily obtained if $A$ separates the points of $K$. The general situation is more complicated, but there is a satisfactory solution if $K$ does not have too many components.

Lemma 5.1. Let $A$ be a subset of $\mathcal{C}(K)$ that contains $\sigma_{1}, \cdots, \sigma_{n}$ and separates the points of $K$. Then there is an open set $U \supset K$ such that $A(U)$ separates the points of $K$.

Proof. If $x \in \sigma(K)$, then $F_{x}=\sigma^{-1}(x) \cap K$ is finite. Hence there is an open set $U_{x} \subset K$ such that $A\left(U_{x}\right)$ separates the points of $F_{x}$. Since $\sigma$ is a local homeomorphism, it follows that $A\left(U_{x}\right)$ separates the points of $F_{y}$ for $y \in \sigma(K)$ sufficiently close to $x$. A compactness argument shows that there are points $x_{1}, \cdots$, $x_{m}$ in $\sigma(K)$ and open sets $V_{1}, \cdots, V_{m}$ covering $\sigma(K)$ such that $A\left(U_{x_{j}}\right)$ separates the points of $F_{y}$ when $y \in V_{j}$. If $U=\bigcap U_{x_{j}}$, it is clear that $A(U) \stackrel{\text { separates the }}{{ }_{j}}$ points of $K$.

Now suppose that $A$ is a closed subalgebra of $\mathcal{C}(K)$ containing $1, \sigma_{1}, \cdots, \sigma_{n}$, closed under differentiation and separating the points of $K$. Choose an open set $U \subset K$ so that $A(U)$ separates the points of $K$. If $V$ is open, $K \subset V \subset U$, then ${ }^{*} U V$ is a homeomorphism of a neighborhood of $K$ in $\Delta A(V)$ onto a neighborhood of $K$ in $\Delta A(U)$. Following the proof of 3.6 we see easily that $A$ is isomorphic to a stable subalgebra of $\mathcal{C}(K)$, considering $K$ as a subset of $\Delta A(U)$.

We now turn to the more general case.

Theorem 5.2. Let $A$ be a closed subalgebra of $\mathcal{O}(K)$ which is closed under differentiation and contains the germs $1, \sigma_{1}, \cdots, \sigma_{n}$. If $K$ bas only finitely many components, then there is a Stein-Riemann domain $S^{\prime}$ with global local coordinate $\sigma^{\prime}$, a compact set $K^{\prime} \subset S^{\prime}$, a closed stable subalgebra $A^{\prime}$ of $\Theta\left(K^{\prime}\right)$ and an isomorphism $\Phi: A \rightarrow A^{\prime}$ such that $\Phi(1)=1, \Phi\left(\sigma_{i}\right)=\sigma_{i}^{\prime}$.

Proof. The technique is similar to that employed in 3.6 and 4.1 , so we omit some details. Choose $x \in \sigma(K)$. We may find an open set $U_{x} \supset K$ such that $A\left(U_{x}\right)$ separates those points of $F_{x}=\sigma^{-1}(x) \cap K$ that are separated by $A$. We assert that $A\left(U_{x}\right)$ separates the points of $F_{y}$ that are separated by $A$ when $y \in \sigma(K)$ is close to $x$. 
If this were not so, we could find sequences $p_{i} \rightarrow p, q_{i} \rightarrow q$ in $K$ such that $\sigma\left(p_{i}\right)=\sigma\left(q_{i}\right), \sigma(p)=\sigma(q)=x, A$ separates $p_{i}$ from $q_{i}$, but $A\left(U_{x}\right)$ does not separate $p_{i}$ from $q_{i}$. Thus $A$ does not separate $p$ from $q$. Since $K$ has only finitely many components, we may assume that all the $p_{i}$ belong to the component $P$, all the $q_{i}$ belong to the component $Q$. If $V$ is an open set containing $K$ and $g \in A(V)$, then $g$ and all its derivatives take the same value at $p$ as at $q$. Thus $g$ identifies a neighborhood of $p$ with a neighborhood of $q$. Connectedness of $P$ and $Q$ implies that $g\left(p_{i}\right)=g\left(q_{i}\right)$ for every $i$. This proves the assertion.

Now a compactness argument shows that we may find an open set $W \supset K$ such that $A(W)$ separates the points of $K$ that are separated by $A$. Then if $W^{\prime}$ is open and $K \subset W^{\prime} \subset W$, it follows that $r_{W W^{\prime}}^{*}$ is a homeomorphism of $r_{W^{\prime}}^{*}(K)$ onto $r_{W}^{*}(K)$. Take $S^{\prime}=\Delta A(W), \sigma^{\prime}=\left(\left(\sigma_{1} \mid W\right)^{\wedge}, \cdots,\left(\sigma_{n} \mid W\right)^{\wedge}\right)$ and $K^{\prime}=r_{W}^{*}(K)$. As in the proof of 3.6, it may be seen that each $\mathbf{f} \in A$ gives rise to a germ $\Phi(f) \in \mathcal{O}\left(r_{W}^{*}(K)\right)$, that $\Phi\left(\sigma_{i}\right)=\sigma_{i}^{\prime}$, and that $\Phi: A \rightarrow \mathcal{Q}\left(r_{W}^{*}(K)\right)$ is an isomorphism onto its range, which is a stable algebra.

It is easy to construct examples showing that 5.2 is false when $K$ has infinitely many components. If we drop the requirement that $\Phi\left(\sigma_{i}\right)=\sigma_{i}^{\prime}$, it seems harder to decide which algebras are isomorphic to stable algebras.

\section{REFERENCES}

1. F. T. Birtel, Some holomorphic function algebras, Summer Gathering on Function Algebras (Aarhus, 1969), Matematik Inst., Aarhus Univ., Aarhus, 1969, pp. 11-18. MR 40 \#7806.

2. - Some aspects of function algebras, Nieuw Arch. Wisk. (3) 17 (1969), 171187. MR $40 \# 7805$.

3. F. T. Birtel and W. R. Zame, Some analytic function algebras, Proc. Sympos. on Several Complex Variables, Park City, Utah, 1970, pp. 1-10.

4. E. Bishop, Holomorphic completions, analytic continuations, and the interpolation of semi-norms, Ann. of Math. (2) 78 (1963), 468-500. MR 27 \#4958.

5. J. E. Bjork, Every compact set in $C^{n}$ is a good compact set, Ann. Inst. Fourier (Grenoble) 20 (1970), fasc. 1, 493-498. MR $41 \# 7154$.

6. N. Bourbaki, Éléments de mathématique. Fasc. XV. Livre V: Espaces vectoriels topologiques, Actualités Sci. Indust., no. 1189, Hermann, Paris, 1953. MR 14, 880.

7. T. W. Gamelin, Uniform algebras, Prentice-Hall, Englewood Cliffs, N. J., 1969.

8. A. Guichardet, Special topics in topological algebras, Gordon and Breach, New York, 1968. MR $39 \# 4673$.

9. R. C. Gunning and H. Rossi, Analytic functions of several complex variables, Prentice-Hall, Englewood Cliffs, N. J., 1965. MR 31 \#4927.

10. R. Harvey and R. O. Wells, Jr., Compact holomorphically convex subsets of a Stein manifold, Trans. Amer. Math. Soc. 136 (1969), 509-516. MR 38 \#3470.

11. L. Van Hove, Topologie des espaces fonctionnels analytiques et des groupes infinis de transformations, Acad. Roy. Belgique Bull. Cl. Sci. (5) 38 (1952), 333-351. MR 14, 287. 
12. W. R. Zame, Stable algebras of holomorphic germs, Dissertation, Tulane University, New Orleans, La., 1970.

13. - Algebras of analytic functions in the plane, Pacific J. Math. (to appear).

14. Jun-iti Nagata, Modern dimension.theory, Bibliotheca Math., vol. 6, Interscience, New York, 1965. MR 34 \#8380.

15. E. Kallin, A nonlocal function algebra, Proc. Nat. Acad. Sci. U.S.A. 49 (1963), 821-824. MR 27 \#2878.

DEPARTMENT OF MATHEMATICS, RICE UNIVERSITY, HOUSTON, TEXAS 77001

Current address: Department of Mathematics, SUNY at Buffalo, Amherst, New York 14226 